\title{
Construction of a transcription factor-long non-coding RNA-microRNA network for the identification of key regulators in lung adenocarcinoma and lung squamous cell carcinoma
}

\author{
SHUAI ZHAO ${ }^{*}$, HONG CHEN $^{1 *}$, BEICHEN DING $^{2}$, JIANING LI $^{1}$, FUZHEN LV $^{1}$, \\ KAIYU HAN ${ }^{1}$, DAN ZHOU ${ }^{1}$, BAIQUAN YU ${ }^{1}$, YAO YU ${ }^{1}$ and WEI ZHANG ${ }^{3}$ \\ ${ }^{1}$ Department of Respiratory Medicine, The Second Affiliated Hospital of Harbin Medical University, Harbin, \\ Heilongjiang 150001; ${ }^{2}$ Department of Urology, Tongji Hospital, Tongji Medical College, \\ Huazhong University of Science and Technology, Wuhan, Hubei 430030; ${ }^{3}$ Department of Respiratory Medicine \\ The First Affiliated Hospital of Harbin Medical University, Harbin, Heilongjiang 150001, P.R. China
}

Received April 14, 2018; Accepted October 23, 2018

DOI: $10.3892 / \mathrm{mmr} .2018 .9769$

\begin{abstract}
The interactions of microRNAs (miRNAs), transcription factors (TFs) and their common target long non-coding RNAs (lncRNAs) can lead to the production of TF-miRNA-lncRNA (TML) network motifs. These motifs are functional regulators that perform a wide range of biological processes, such as carcinogenesis. However, TML network motifs have not been systematically identified, and their roles in lung adenocarcinoma (LUAD) and lung squamous carcinoma (LUSC) are largely unknown. In the present study, a computational integration approach was performed using multiple sources in order to construct a global TML network for LUAD and LUSC. The analysis revealed several dysregulated TML network motifs, which were common between the two lung cancer subtypes or specific to a single cancer subtype. In addition, functional analysis further indicated that the TML network motifs may potentially serve as putative biomarkers in LUAD and LUSC. The associations between drug treatments and dysregulated TML network motifs were also examined. Collectively, the present study elucidated the roles of TML network motifs in LUAD and LUSC, which may be beneficial for understanding the pathogenesis of lung cancer and its potential treatment.
\end{abstract}

Correspondence to: Professor Wei Zhang, Department of Respiratory Medicine, The First Affiliated Hospital of Harbin Medical University, 23 Youzheng Road, Harbin, Heilongjiang 150001, P.R. China

E-mail: weipoza@163.com

${ }^{*}$ Contributed equally

Key words: long non-coding RNA, lung cancer, topological feature, biomarker, drug target

\section{Introduction}

Lung cancer is a common type of cancer and the main cause of cancer-associated mortality worldwide $(1,2)$. The classification of lung cancer is divided into two major categories, namely small cell lung cancer and non-small cell lung cancer (NSCLC) (3). The two main histological types of NSCLC are lung adenocarcinoma (LUAD) and lung squamous carcinoma (LUSC). It has been shown that the prognosis of LUAD patients is worse compared with that of LUSC (4). In the majority of cases, the type of cancer (LUAD or LUSC) is distinguished based on standard morphological criteria; however, the definition of glandular and/or squamous carcinoma features is subtle and/or localized to the tissue area. Thus, it is difficult to distinguish between these two types of cancer in several poorly differentiated tumors.

Previous studies have focused on the genome, epigenome, transcriptome and proteome of lung cancer in order to identify new cancer-driven factors that may be targeted clinically (5-7). In the last decade, researchers have found that the changes noted in certain transcriptional regulators can significantly improve the overall survival rate of a small subset of patients with lung cancer $(8,9)$. Transcriptional and post-transcriptional levels are included in transcriptional regulators. Since transcription factors (TFs) are essential for the regulation of gene expression, they are present in all living organisms. Small non-coding RNA molecules containing approximately 22 nucleotides, termed microRNAs (miRNAs), have been identified in plants, animals and certain viruses, and are involved in RNA silencing and post-transcriptional regulation of gene expression (10). In addition, long-non-coding RNAs (lncRNAs) with a length of $>200$ nucleotides are distributed throughout the genome, and the majority of human diseases, including cancer, are associated with lncRNA expression disorders $(11,12)$. A number of studies have also reported that the associations between TFs, lncRNAs and miRNAs may serve a role in lung cancer development. For instance, a recent study constructed a TF-lncRNA-gene network to illustrate how TFs regulate lncRNA and gene expression in LUAD (13). 
Another study systematically analyzed the interaction of co-expressed mRNAs, miRNAs and lncRNAs activated by transforming growth factor- $\beta 1$, and detected a total of 24 mRNAs, 11 miRNAs and 33 lncRNAs that interacted with one another (14). Therefore, the regulatory network information of TFs, miRNAs and lncRNAs can be utilized in order to reveal disease-modulating genes and pathways, and to identify new therapeutic targets. In particular, TFs and miRNAs can coordinate to regulate common target genes (15), while transcription of miRNAs is regulated by TFs, and a common set of target lncRNAs are regulated by both TFs and miRNAs. This type of motif is defined as a TF-miRNA-lncRNA (TML) network motif. However, it is insufficient to analyze the difference between LUAD and LUSC at the molecular level based on the application of regulatory network motifs.

In the present study, the TML network motif was defined as a motif that includes a TF, miRNA and lncRNA, in which the TF regulates the expression of the miRNA, and both the TF and miRNA regulate a common set of target lncRNAs. A global TML network was constructed, and then its features were characterized, and a computational approach was designed by integrating the interaction and expression data of miRNAs, TFs and IncRNAs. Dysregulated TML network motifs that were common and specific with regard to LUAD and LUSC were identified. The dysregulated TML network motifs were associated with cancer-related functions and pathways. It is important to note that several miRNAs in these TML network motifs may be potential drug targets. The results of the present study elucidated the roles of TML network motifs in LUAD and LUSC, which may be beneficial for understanding lung cancer pathogenesis and treatment. The applications of these findings require further investigation in future studies.

\section{Materials and methods}

Collecting high-throughput expression data for miRNAs, TFs and lncRNAs. The expression data of lncRNAs, TFs and miRNAs were obtained from LUSC and LUAD tissues and their corresponding adjacent normal tissues as previously described (16). The raw read counts for each exon were downloaded from The Cancer Genome Atlas (TCGA) (https://portal.gdc.cancer.gov/) level 3 dataset, and the human TF and lncRNA annotations from GENCODE were used to map these exons (17). In order to obtain expression data of human TFs and lncRNAs, the reads per kilobase of transcript per million mapped reads were recalculated for the TFs and lncRNAs. miRNA sequencing data (Illumina HiSeq miRNA Seq; Illumina, Inc., San Diego, CA, USA) of LUAD and LUSC were also downloaded from TCGA (level 3) (7). Finally, a total of 166 LUAD samples and 9 matched normal samples were selected, whereas 120 LUSC samples and 9 matched normal samples were selected.

Establishing a genome-wide TML network. TML network motifs comprise a TF, an miRNA and their common target lncRNAs. In these motifs, the TF strictly regulates the expression of the miRNA, and these two cooperatively regulate a common set of target lncRNAs. Three types of regulatory interactions are required in order to construct a global TML network, including miRNA-lncRNA, TF-miRNA and
TF-lncRNA interactions. In the present study, the CLIP-Seq experimentally supported miRNA-lncRNA interaction data were downloaded from starBase version 2.0 (18). In addition, the SNP@lincTFBS database provided global TF binding sites for lncRNAs, and these data were used for the TF-lncRNA regulatory interactions (19). Finally, a large number of experimental data on TF-miRNA interactions were extracted from the TransmiR database by literature and publication searches (20).

Dissecting topological features for the TML network. The total TML network features were obtained by four measurements, as follows: Degrees, topological coefficient, connectivity and clustering coefficient of nodes.

Identifying dysregulated TML motifs in LUAD and LUSC. A comprehensive pipeline was subsequently established in order to identify dysregulated TML network motifs in LUAD and LUSC, based on the integration of the TML network and the expression profiling of the data. Initially, a Student's t-test was performed, and the derived P-values were used to evaluate the differences in TF, IncRNA and miRNA expression levels between the lung cancer samples and the corresponding normal samples in each single TML network motif. Each interaction pair (TF-lncRNA, TF-miRNA and miRNA-lncRNA regulatory interactions) in the TML network motif, the study calculated the Pearson correlation coefficients (PCCs) and the levels of difference between these coefficients with regard to the lung cancer and normal samples. The associations between regulatory interactions were represented by the absolute differences of PCCs between lung cancer and normal samples. Subsequently, the differential expression P-values and PCCs were merged in order to calculate two comprehensive scores (namely Score $_{\text {dif }}$ and Score $_{\text {pcc }}$ ) for TML as follows:

$$
\begin{gathered}
\text { Score }_{\text {dif }}=P_{T F} P_{\text {miRNA }} P_{\text {lncRNA }} \\
\text { Score }_{p c c}=\left|\left(L_{T m}-N_{T m}\right)\left(L_{T l}-N_{T l}\right)\left(L_{m l}-N_{m}\right)\right|
\end{gathered}
$$

where $\mathrm{P}_{\mathrm{TF}}, \mathrm{P}_{\text {miRNA }}$ and $\mathrm{P}_{\text {IncRNA }}$ represent the differential expression P-values of TF, miRNA and lncRNA, respectively, in

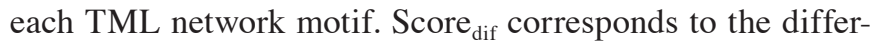
ence in expression of a TML network motif between the lung cancer and the normal samples. $\mathrm{L}_{\mathrm{Tm}}, \mathrm{L}_{\mathrm{Tl}}$ and $\mathrm{L}_{\mathrm{ml}}$ correspond to the PCCs of the three regulatory regulations, namely the TF-miRNA, TF-lncRNA and miRNA-lncRNA interaction pairs, respectively, for the lung cancer samples. $\mathrm{N}_{\mathrm{Tm}}, \mathrm{N}_{\mathrm{Tl}}$, and $\mathrm{N}_{\mathrm{ml}}$ represent the PCCs for the TF and miRNA, TF and lncRNA, and miRNA and lncRNA pairs, respectively, for the control samples. Score ${ }_{\mathrm{pcc}}$ is the absolute distinction of the PCC score between the lung cancer and control samples in the total TML motif.

An equally-weighted multidimensional approach was then used to rank all the TML network motifs for lung cancer based

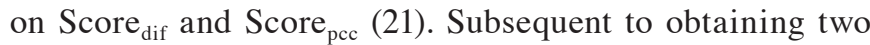
ranked lists based on the two aforementioned scores respectively, the ranking position of the two lists was integrated to calculate the final ranking score for each TML network motif. The higher ranking score represented higher dysregulated motif levels in lung cancer. Furthermore, each final motif 


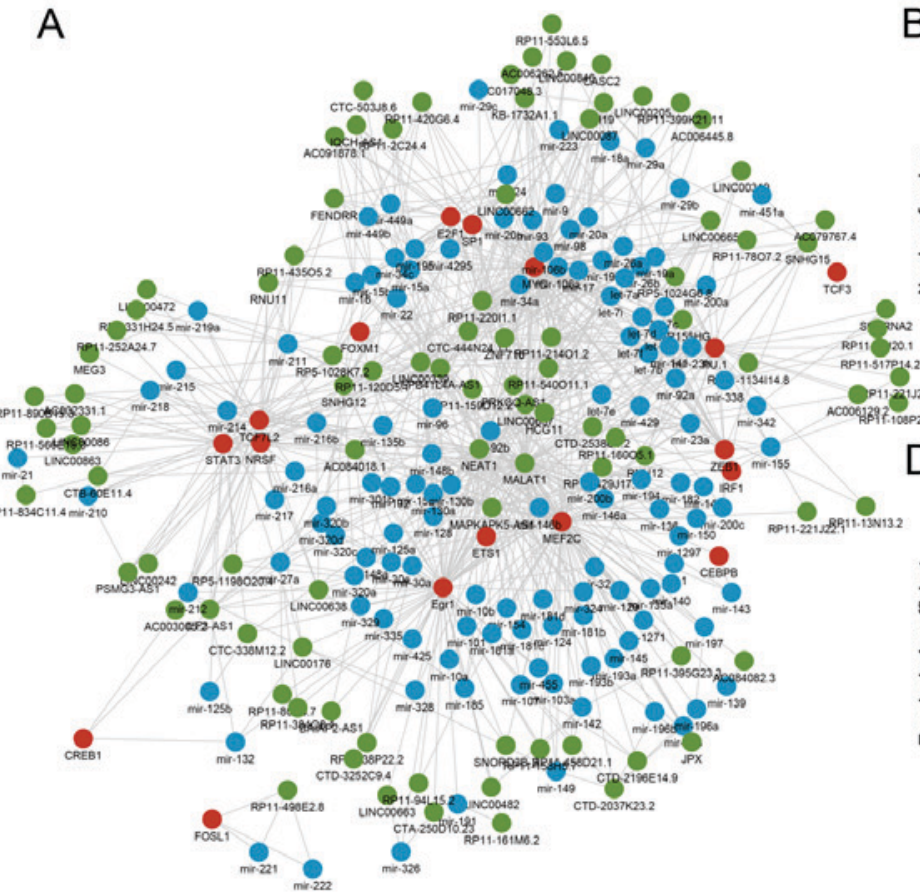

B
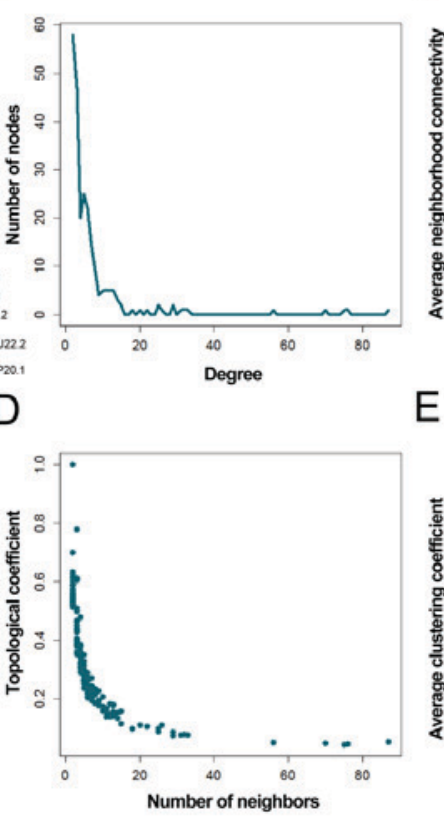

C

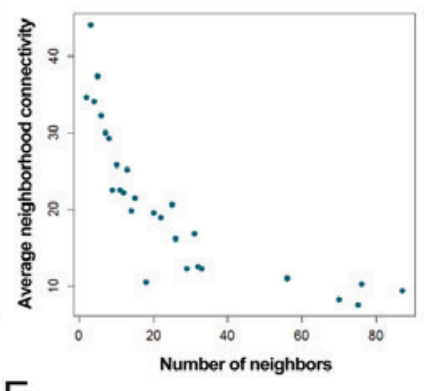

E

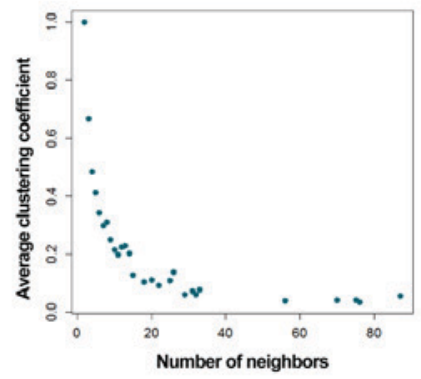

Figure 1. Global topological characteristics of the TML network. (A) Global TML network, including TFs (red), miRNAs (blue) and lncRNAs (green). Basic characteristics of the network are displayed, including (B) degree, (C) connectivity, (D) topological coefficient and (E) clustering coefficient of TFs, miRNAs and lncRNAs. TML, TF-miRNA-lncRNA; miRNA, microRNA; TF, transcription factor; lncRNA, long non-coding RNA.

ranking score was compared with the permutation-based score list, which was generated by randomly disturbing all sample labels in the expression profile for 1,000 times. This was used to produce a significant P-value for each TML motif. Finally, the significant dysregulated TML motifs were obtained for LUAD and LUSC $(\mathrm{P}<0.05)$.

Gene set enrichment analysis. IncRNAs from dysregulated TML network motifs were used to perform functional enrichment using default parameters on the Enrichr tool online web server (22). Enriched Gene Ontology (GO) terms were obtained that were selected at a P-value of $<0.05$, which indicated a statistically significant difference.

The drug targets analyses for dysregulated TML network motifs. We determined the associations between drugs and miRNAs using SM2miR (23), a database of the experimentally validated small molecules that affect the expression of miRNAs. Then, a drug-miRNA network for miRNAs in dysregulated TML network motifs was generated based on data from SM2miR.

\section{Results}

Topological characteristics of the TML network. Multiple data sources were integrated in order to identify $>600$ TML network motifs. Each motif consisted of a TF, an miRNA and their common target lncRNAs. A global TML network containing 240 nodes (96 lncRNAs, 17 TFs and 127 miRNAs) and 878 edges was constructed (Fig. 1A). As expected, this transcriptional regulatory network was similar to the scale-free network topology (Fig. 1B), which has a degree distribution that follows a power law, at least asymptotically.
In addition, the connectivity, topological coefficient and clustering coefficient of nodes were computed and analyzed. All the aforementioned analyzed characteristics indicated scale-free distributions (Fig. 1C-E), suggesting that the TML network was a small-world network (24). The mean level of the neighborhood connectivity with $\mathrm{k}$ neighbors was defined as the neighborhood connectivity distribution $(\mathrm{k}=0,1 \ldots \mathrm{n})$. The network highlighted a hierarchical modularity phenomenon due to the stepwise increase of the degrees of connectivity following the topological coefficient decrease.

Specific TML motifs are significantly dysregulated in LUAD and LUSC. Dysregulated TML network motifs that were significantly different with regard to the LUAD and LUSC in the TML network were identified, and the functional significance of the TML network motifs was further analyzed. These dysregulated motifs were used to construct significantly dysregulated TML sub-networks for LUAD and LUSC (Fig. 2). A total of 11 TML network motifs, including 7 lncRNAs, 5 TFs and 8 miRNAs, were obtained in LUAD (Fig. 2A), while a total of 15 TML network motifs with 8 lncRNAs, 5 TFs and 13 miRNAs were obtained in LUSC (Fig. 2B). In these dysregulated TML network motifs, a number of specific TFs, miRNAs and lncRNAs have been reported to serve essential roles in lung cancer. For instance, a previous study reported that mutation of the zinc finger protein family member 718 may be a potential germline mutation of lung cancer (25). In addition, let-7c, let-7d and let-7f were all present in dysregulated TML motifs in the present analysis, whereas it has been reported that the let-7 miRNA family suppressed NSCLC development (26). Notably, a study demonstrated that the MYC-regulated long non-coding RNA H19 was associated with poor prognosis 

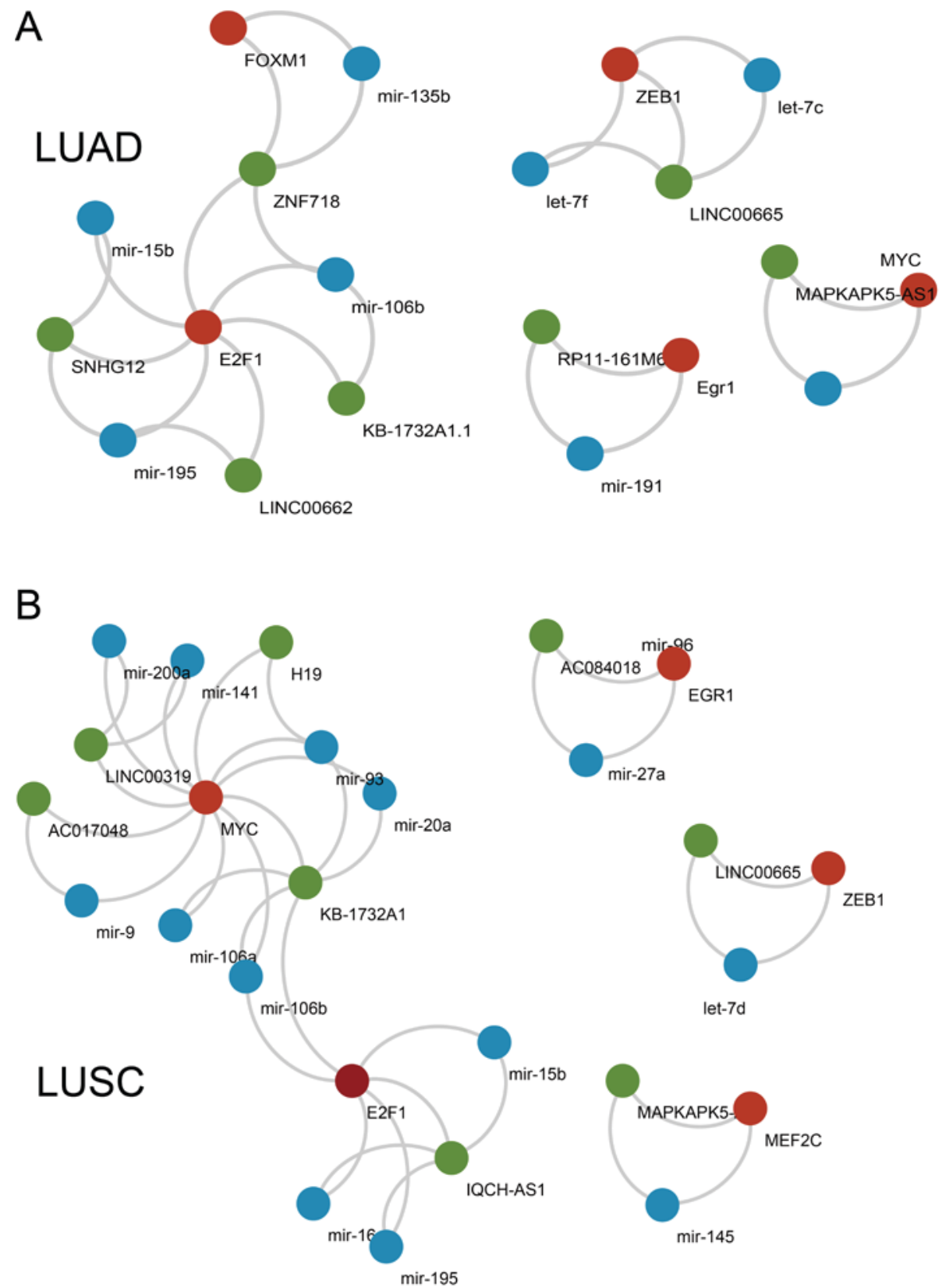

(A) displayed. TFs, miRNAs and IncRNAs are colored red, blue and green, respectively. TML, TF-miRNA-lncRNA; miRNA, microRNA; TF, transcription factor; lncRNA, long non-coding RNA; LUAD, lung adenocarcinoma; LUSC, lung squamous carcinoma.

and affected cell proliferation in NSCLC (27). Similarly, this association was also identified in the present analysis.

Common TML motifs between LUAD and LUSC reveal a lung cancer subtype-specific mechanism. The performance of the TML network motif in distinguishing lung cancer subtypes was investigated in the present study. LUAD and LUSC are two major histological subtypes of NSCLC. Specific differences were apparent with regard to the underlying mechanisms, which suggested the association of specific TMLs with LUAD and LUSC. Two subnetworks of significant TML network motifs in LUAD and LUSC were integrated, and 10 common factors were detected between the two lung cancer subtypes, including 4 TFs, 3 miRNAs and 3 lncRNAs (Fig. 3A and B). For instance, the TF E2F1,
IncRNA KB-1732A1.1 and miR-15b were identified in LUAD and LUSC. Several of these common factors were reported to be associated with lung cancer. Recently, it was reported that mutations in the tyrosine kinase domain of the epidermal growth factor receptor (EGFR) gene occurred in a subset of patients with lung cancer, indicating a marked response to treatment by EGFR tyrosine kinase inhibitors (28). A previous study revealed that miR-195 was significantly downregulated in NSCLC samples and cell lines compared with the corresponding normal counterparts, and that it functioned as a tumor suppressor (29).

In addition, each lung cancer subtype contained its own specific factors. For instance, FOXM1 may serve an important role in advancing LUAD progression. Aberrant FOXM1 expression directly and constitutively activates SNAIL, thereby 

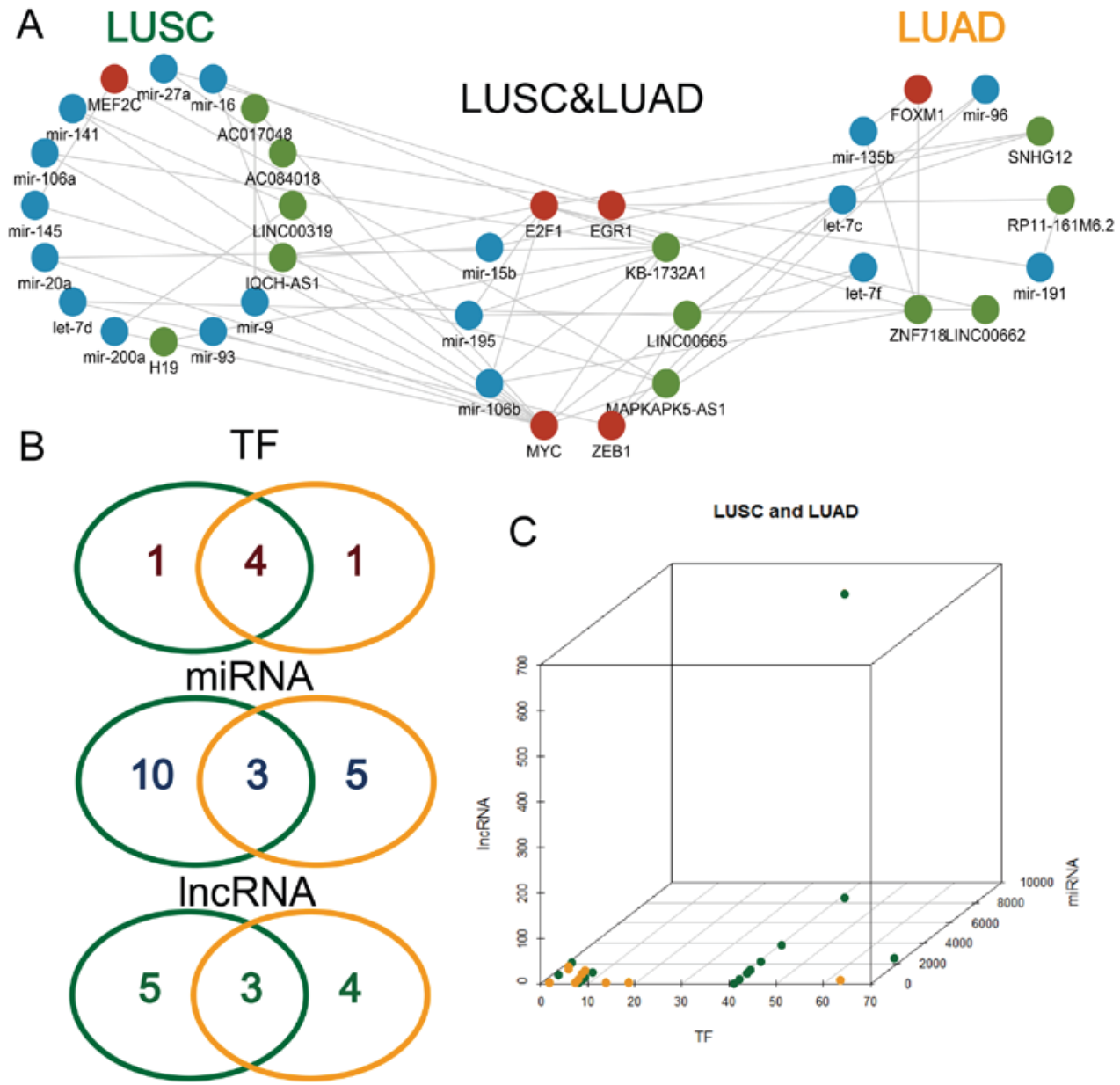

Figure 3. Common and specific TML network motifs in LUAD and LUSC. (A) Sub-network of dysregulated TML network motifs in LUSC, LUAD and the intersection of LUAD and LUSC. (B) Venn diagram denoting the intersection of TFs, miRNAs and lncRNAs between LUAD and LUSC. Six motifs are shown as examples. (C) The cube represents three expression layers of lncRNAs, miRNAs and TFs in LUAD and LUSC. Yellow nodes represent dysregulated TML network motifs in LUAD samples and green nodes represent a dysregulated TML network motif in LUSC samples. The expression values of TFs, miRNAs and lncRNAs are shown along the $\mathrm{x}, \mathrm{y}$ and $\mathrm{z}$ axes, respectively. TML, TF-miRNA-lncRNA; miRNA, microRNA; TF, transcription factor; lncRNA, long non-coding RNA; LUAD, lung adenocarcinoma; LUSC, lung squamous carcinoma.

promoting LUAD metastasis. Inhibition of FOXM1-SNAIL signaling may thus present an ideal target for future cancer treatment (30).

The data also indicated that certain TML network motifs may distinguish LUAD and LUSC samples by combining multiple layers of TML network motif's expression (Fig. 3C). A TML network motif was recognized as a node in three-dimensional space. The $\mathrm{z}-, \mathrm{y}$ - and $\mathrm{x}$-axes dimensions respectively represented the expression of IncRNA, miRNA and TF in a TML network motif. It was observed that a single layer of expression, including miRNA, IncRNA or TF, was unable to distinguish the LUAD and LUSC samples. However, the TML network motifs were able to effectively distinguish between the two different cancer subtypes by combining multiple layers of expression. The results also indicated that the nodes of LUAD and LUSC samples could clearly be separated in three-dimensional space. Overall, the TML motif separated LUSC and LUAD samples more efficiently as compared with the use of each single type of molecules.

Functional analysis demonstrates the roles of TML motifs in LUAD and LUSC. GO analysis based on the IncRNAs in the dysregulated TML network motifs of LUAD and LUSC was also performed in the present study. These dysregulated lncRNAs were enriched in various GO terms, including negative regulation of lipoprotein lipase activity, regulation of translation and lncRNA-mediated regulation of translational elongation (Fig. 4A and B). The function termed positive regulation of protection from non-homologous end joining at the telomere sites was also identified as a significant pathway. Telomerase is an attractive cancer target as it appears to be required in essentially all tumors for immortalization of a subset of cells, such as cancer stem cells (31).

In addition, two dysregulated factors, namely MAPKAPK5 and MYC, were identified as key genes in the p38 mitogen-activated protein kinase (MAPK) signaling pathway in the current study (Fig. 4C). Uncontrolled growth is a necessary step for the development of cancer. In various types of cancer, a defect in the MAPK signaling pathway leads to this uncontrolled growth. Several compounds can inhibit key proteins in the MAPK pathway, and these may serve as potential drugs for the treatment of cancer $(32,33)$. Taken together, functional analysis indicated that the dysregulated TML network motifs identified by our method exhibited a strong association with cancer. 

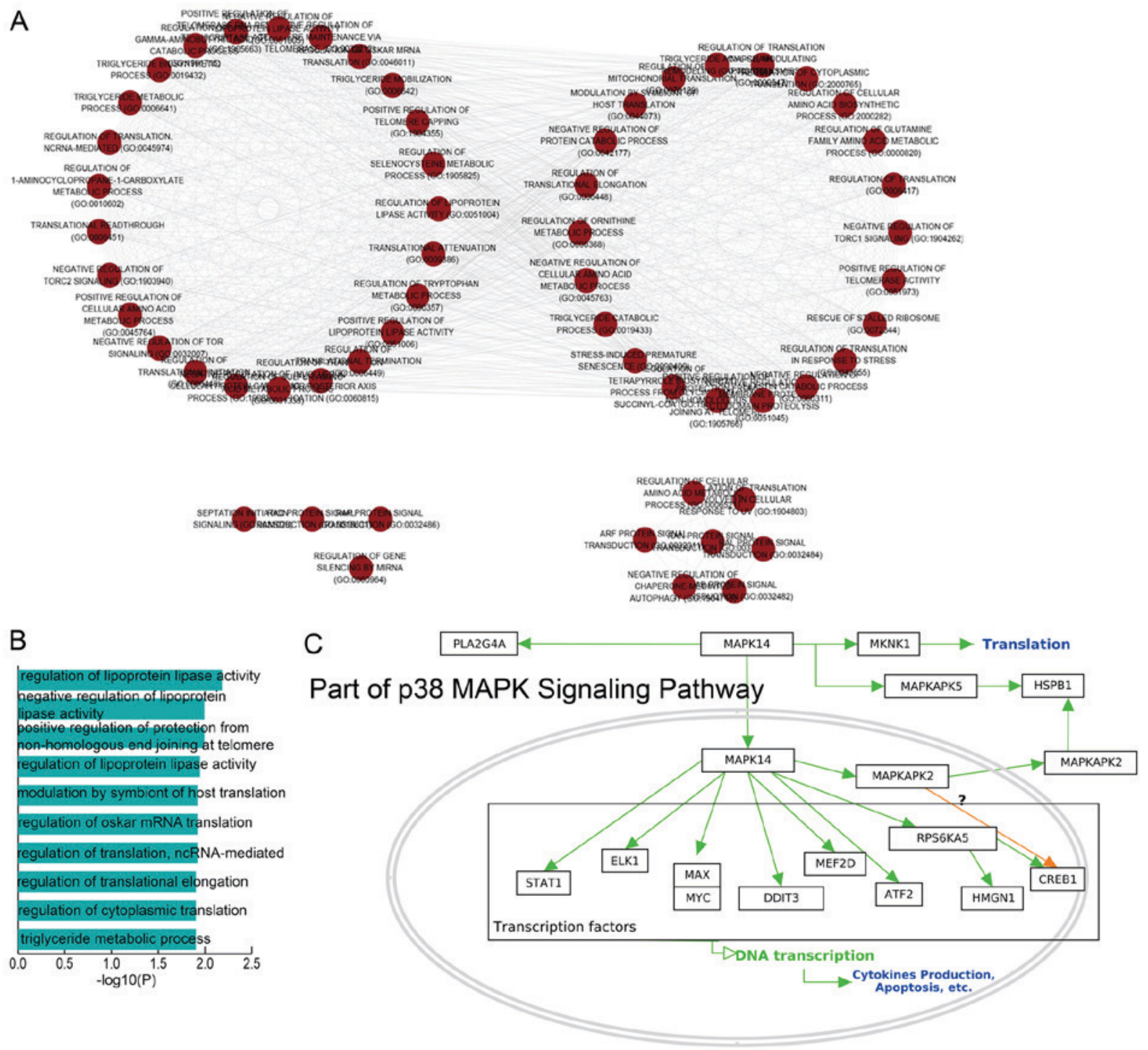

Figure 4. Functional analysis of long non-coding RNAs in dysregulated TML network motifs. (A) Functional enrichment map of GO terms. Each node represents a GO term, and is grouped and annotated by GO similarity. The node size represents the number of genes in each term, while links represent the overlap of shared genes between connecting GO terms. (B) Bar plot of GO terms. (C) p38 MAPK signaling pathway. Two dysregulated factors, namely MAPKAPK5 and MYC, were identified as key genes in this pathway. TML, transcription factor-microRNA-long non-coding RNA; GO, Gene Ontology; MAPKAPK5, mitogen-activated protein kinase-activated protein kinase 5 .

TML network motifs contain potential drug targets. A cancer-associated drug-miRNA network based on miRNAs in dysregulated TML network motifs was constructed, and the data from SM2miR were used (33). SM2miR is a database of the experimentally validated small molecules that affect the expression of miRNAs (23). The cancer-associated drug-miRNA network included 68 nodes (24 miRNAs and 44 drugs) and 118 edges (Fig. 5A). Initially, the common miRNAs in LUAD and LUSC, including miR-15b, miR-106b and miR-195, were analyzed (Fig. 5B). These three miRNAs were all associated with the drug 5-fluorouracil, which is widely used in the treatment of cancer, including colorectal and breast cancer (34). Although the miRNAs identified in the dysregulated TML network motifs were influenced by cancer drug treatment, the regulated direction (upregulated or downregulated) was distinct and indicated that the drug effect to the miRNAs was complex. In addition, certain drugs were associated with miR-15b, such as etoposide, which is a chemotherapy medication used for the treatment of a number of cancer types, including lung cancer (35). Another such compound is ginsenoside, a traditional Chinese medicine that exhibited an inhibitory effect on the cell growth of various cancer cells and animal models, which was observed to be associated with miR-15b and miR-106b in the current study (Fig. 5C) (36). Furthermore, the current results identified a number of lung cancer treatment drugs, including gemcitabine, cisplatin and vincristine. Gemcitabine, which is used in the treatment of NSCLC, was associated with the let-7 miRNA family, including let-7c, let-7d and let-7f. Taken together, the data of the current study indicated that the dysregulated TML network motifs may be used to identify drug targets for novel treatment strategies.

\section{Discussion}

Diverse types of regulatory transcripts (such as lncRNAs, TFs and miRNAs) exhibit different types of interactions. Recently, 
A

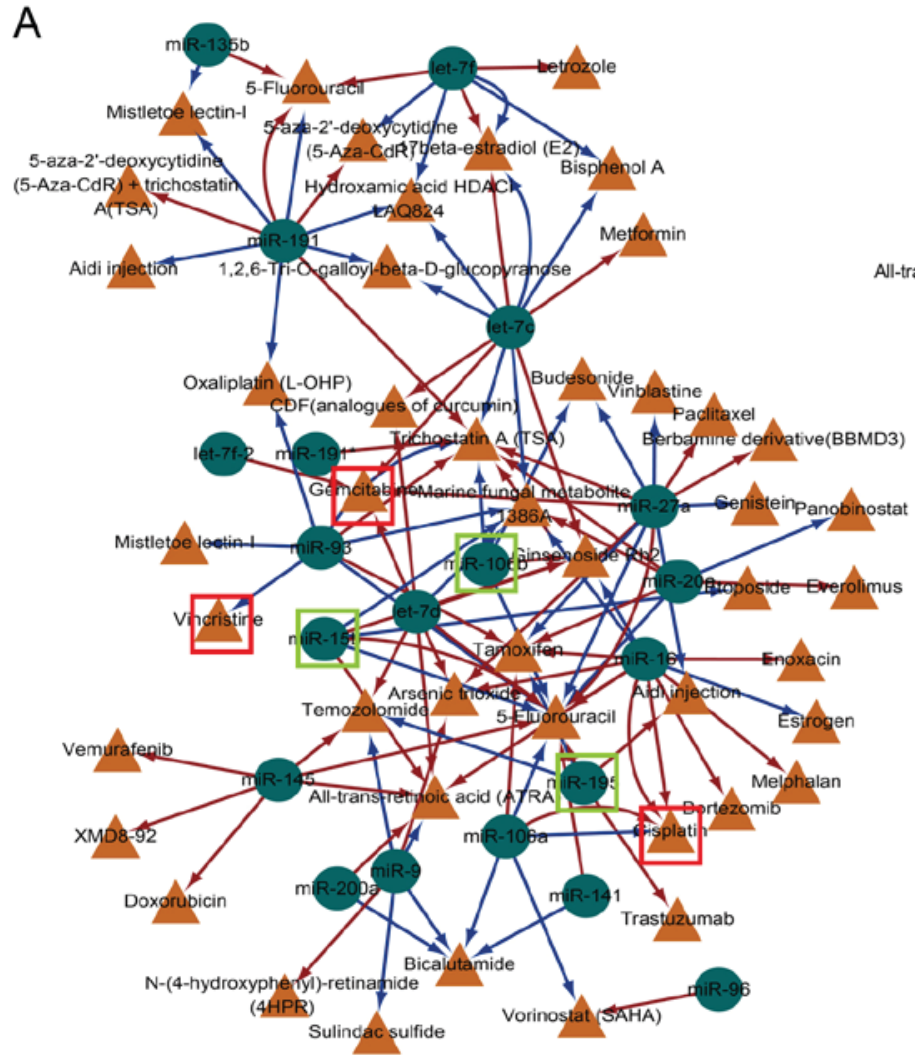

B

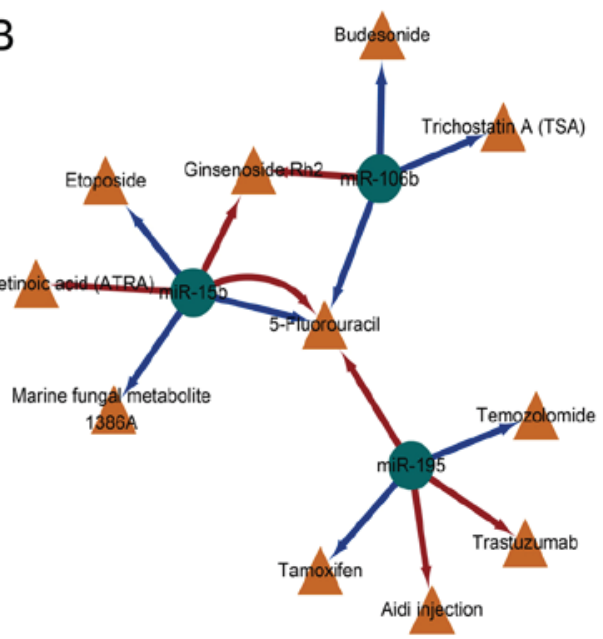

C

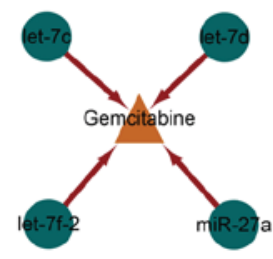

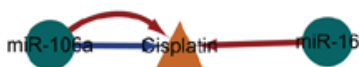

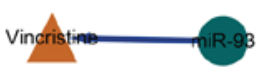

Figure 5. Anti-cancer drug effects of miRNAs in dysregulated TML network motifs involved in LUAD and LUSC. (A) Network of anti-cancer drugs and miRNAs in dysregulated TML motifs in LUAD and LUSC. Blue circular nodes represent the miRNAs, orange triangles represent the anti-cancer drugs, blue edges represent downregulation of miRNAs and red edges represent upregulation of miRNAs. (B) Sub-network of common miRNAs and anti-cancer drugs in LUAD and LUSC. (C) Sub-network of certain clinically approved lung cancer drugs and specific miRNAs. TML, transcription factor-miRNA-long non-coding RNA; miRNA, microRNA; LUAD, lung adenocarcinoma; LUSC, lung squamous carcinoma.

the application of computational modeling has been employed for the prediction of lung cancer incidence. Certain miRNAs and IncRNAs have been identified as potential biomarkers, and interact with each other in lung cancer $(37,38)$. These different types of molecules form complex network motifs in order to play specific biological roles and accordingly affect the pathogenic mechanisms in several cancer types. Other types of TML motifs have also been identified, such as motifs that describe the regulatory action of miRNAs and lncRNAs on TFs or the regulatory action of IncRNAs on miRNAs (39). The present study concentrated on an important network motif, namely the TML network motif, which included a TF, miRNA and lncRNA. A computational pipeline was presented in order to study the TML network motifs by integrating the interaction and expression data of TFs, miRNAs and lncRNAs. The study identified dysregulated TML network motifs in the two lung cancer types, LUAD and LUSC. Furthermore, the results as to whether a TML motif i specific in one type of cancer or common in both, were consistent with previous studies on the tissue specificity of lncRNAs and miRNAs. Cancer subtype-specific TML network motifs may aid the development of drugs that minimize the side effects of patient treatment. Functional and drug effect analyses conducted in the present study suggested that certain TML network motifs may serve as putative biomarkers for LUAD and LUSC. The data presented in the current study are in agreement with previous studies that highlighted the use of single miRNAs and lncRNAs as disease biomarkers, since the TML network motif identified may be considered as a significant pattern to study lung cancer.

In the present study, the clinical applications of dysregulated TML network motifs were further explored for drug development. The data revealed that the miRNAs in LUAD and LUSC-associated TMLs were influenced by anticancer drug treatment. The TML motifs may act as functional modules, providing the potential mechanism of action of the corresponding drugs. Furthermore, the results indicated that the same miRNAs had distinct regulatory mechanisms with regard to the different drugs. For example, Let-7c was up-regulated by Metformin and downregulated by Trichostatin A. Similarly, the same drugs may also influence different miRNA expression levels by distinct directions. Further studies should explore the multiple changes in the transcriptome that occur as a result of the treatment of the patients with anti-cancer drugs.

In conclusion, the results of the present study provided novel insights into the potential function of miRNAs, lncRNAs and TFs in LUAD and LUSC. Dysregulated TML network motifs and the common motifs in LUAD and LUSC were identified. The common features between LUAD and LUSC indicated that diverse mechanisms were involved and different clinical treatments may be suitable for the two cancer subtypes. Functional and drug analyses further indicated the fundamental action of the TML network motifs in lung cancer. Taken together, the present study identified and investigated 
TML network motifs, and the obtained data provide further insight into the multi-level crosstalk regulation found in LUAD and LUSC.

\section{Acknowledgements}

Not applicable.

\section{Funding}

This study was supported by the Research Fund of the First Affiliated Hospital of Harbin Medical University (grant no. 2017Y013).

\section{Availability of data and materials}

All data generated or analyzed during this study are included in this published article.

\section{Authors' contributions}

SZ, HC and WZ conceived and designed the present study. SZ, $\mathrm{BD}, \mathrm{JL}, \mathrm{FL}$ and $\mathrm{KH}$ performed the experiment and analyzed the data. DZ, BY and YY verified and improved the computational approaches conducted in the present study. HC and SZ were involved in drafting the manuscript. All authors read and approved the final manuscript.

\section{Ethics approval and consent to participate}

Not applicable.

\section{Patient consent for publication}

Not applicable.

\section{Competing interests}

The authors declare that they have no competing interests.

\section{References}

1. Saracci R and Wild CP: Fifty years of the international agency for research on cancer (1965 to 2015). Int J Cancer 138: 1309-1311, 2016

2. Inamura K, Fujiwara T, Hoshida Y, Isagawa T, Jones MH, Virtanen C, Shimane M, Satoh Y, Okumura S, Nakagawa K, et al: Two subclasses of lung squamous cell carcinoma with different gene expression profiles and prognosis identified by hierarchical clustering and non-negative matrix factorization. Oncogene 24 7105-7113, 2005

3. Li S, Huang S and Peng SB: Overexpression of G protein-coupled receptors in cancer cells: Involvement in tumor progression. Int J Oncol 27: 1329-1339, 2005.

4. Suzuki K, Nagai K, Yoshida J, Nishimura M, Takahashi K, Yokose T and Nishiwaki Y: Conventional clinicopathologic prognostic factors in surgically resected nonsmall cell lung carcinoma. A comparison of prognostic factors for each pathologic TNM stage based on multivariate analyses. Cancer 86: 1976-1984, 1999.

5. Cancer Genome Atlas Research Network: Comprehensive molecular profiling of lung adenocarcinoma. Nature 511: 543-550, 2014.

6. Imielinski M, Berger AH, Hammerman PS, Hernandez B Pugh TJ, Hodis E, Cho J, Suh J, Capelletti M, Sivachenko A, et al: Mapping the hallmarks of lung adenocarcinoma with massively parallel sequencing. Cell 150: 1107-1120, 2012.
7. Cancer Genome Atlas Research Network: Comprehensive genomic characterization of squamous cell lung cancers. Nature 489: 519-525, 2012.

8. Shaw AT, Kim DW, Nakagawa K, Seto T, Crinó L, Ahn MJ, De Pas T, Besse B, Solomon BJ, Blackhall F, et al: Crizotinib versus chemotherapy in advanced ALK-positive lung cancer. N Engl J Med 368: 2385-2394, 2013.

9. Mok TS, Wu YL, Thongprasert S, Yang CH, Chu DT, Saijo N, Sunpaweravong P, Han B, Margono B, Ichinose Y, et al: Gefitinib or carboplatin-paclitaxel in pulmonary adenocarcinoma. N Engl J Med 361: 947-957, 2009.

10. Ambros V: The functions of animal microRNAs. Nature 431: 350-355, 2004.

11. St Laurent G, Wahlestedt C and Kapranov P: The landscape of long noncoding RNA classification. Trends Genet 31: 239-251, 2015.

12. Bassett AR, Akhtar A, Barlow DP, Bird AP, Brockdorff N, Duboule D, Ephrussi A, Ferguson-Smith AC, Gingeras TR, Haerty W, et al: Considerations when investigating lncRNA function in vivo. Elife 3: e03058, 2014.

13. Qiu M, Xu Y, Wang J, Zhang E, Sun M, Zheng Y, Li M, Xia W, Feng D, Yin R and Xu L: A novel lncRNA, LUADT1, promotes lung adenocarcinoma proliferation via the epigenetic suppression of p27. Cell Death Dis 6: e1858, 2015.

14. Liu H, Zhao X, Xiang J, Zhang J, Meng C, Zhang J, Li M, Song X and Lv C: Interaction network of coexpressed mRNA, miRNA, and $\operatorname{lncRNA}$ activated by TGF- $\beta 1$ regulates EMT in human pulmonary epithelial cell. Mol Med Rep 16: 8045-8054, 2017.

15. Shalgi R, Lieber D, Oren M and Pilpel Y: Global and local architecture of the mammalian microRNA-transcription factor regulatory network. PLoS Comput Biol 3: e131, 2007.

16. Wang P, Ning S, Zhang Y, Li R, Ye J, Zhao Z, Zhi H, Wang T, Guo Z and Li X: Identification of lncRNA-associated competing triplets reveals global patterns and prognostic markers for cancer. Nucleic Acids Res 43: 3478-3489, 2015.

17. Kersey PJ, Lawson D, Birney E, Derwent PS, Haimel M, Herrero J, Keenan S, Kerhornou A, Koscielny G, Kähäri A, et al: Ensembl Genomes: extending Ensembl across the taxonomic space. Nucleic Acids Res 38 (Database Issue): D563-D569, 2010.

18. Li JH, Liu S, Zhou H, Qu LH and Yang JH: starBase v2.0: Decoding miRNA-ceRNA, miRNA-ncRNA and protein-RNA interaction networks from large-scale CLIP-Seq data. Nucleic Acids Res 42 (Database Issue): D92-D97, 2014.

19. Ning S, Zhao Z, Ye J, Wang P, Zhi H, Li R, Wang T, Wang J, Wang L and Li X: SNP@lincTFBS: An integrated database of polymorphisms in human LincRNA transcription factor binding sites. PLoS One 9: e103851, 2014.

20. Wang J, Lu M, Qiu C and Cui Q: TransmiR: A transcription factor-microRNA regulation database. Nucleic Acids Res 38 (Database Issue): D119-D122, 2010.

21. Aerts S, Lambrechts D, Maity S, Van Loo P, Coessens B, De Smet F, Tranchevent LC, De Moor B, Marynen P, Hassan B, et al: Gene prioritization through genomic data fusion. Nat Biotechnol 24: 537-544, 2006.

22. Kuleshov MV, Jones MR, Rouillard AD, Fernandez NF, Duan Q Wang Z, Koplev S, Jenkins SL, Jagodnik KM, Lachmann A, et al: Enrichr: A comprehensive gene set enrichment analysis web server 2016 update. Nucleic Acids Res 44: W90-W97, 2016.

23. Liu X, Wang S, Meng F, Wang J, Zhang Y, Dai E, Yu X, Li X and Jiang W: SM2miR: A database of the experimentally validated small molecules' effects on microRNA expression. Bioinformatics 29: 409-411, 2013.

24. Amaral LA, Scala A, Barthelemy M and Stanley HE: Classes of small-world networks. Proc Natl Acad Sci USA 97: 11149-11152, 2000.

25. Kanwal M, Ding XJ, Ma ZH, Li LW, Wang P, Chen Y, Huang YC and Cao Y: Characterization of germline mutations in familial lung cancer from the Chinese population. Gene 641: 94-104, 2018.

26. Kumar MS, Erkeland SJ, Pester RE, Chen CY, Ebert MS, Sharp PA and Jacks T: Suppression of non-small cell lung tumor development by the let-7 microRNA family. Proc Natl Acad Sci USA 105: 3903-3908, 2008.

27. Zhang E, Li W, Yin D, De W, Zhu L, Sun S and Han L: c-Myc-regulated long non-coding RNA H19 indicates a poor prognosis and affects cell proliferation in non-small-cell lung cancer. Tumour Biol 37: 4007-4015, 2016.

28. Kosaka T, Yatabe Y, Endoh H, Kuwano H, Takahashi T and Mitsudomi T: Mutations of the epidermal growth factor receptor gene in lung cancer: Biological and clinical implications. Cancer Res 64: 8919-8923, 2004. 
29. Yongchun Z, Linwei T, Xicai W, Lianhua Y, Guangqiang Z Ming Y, Guanjian L, Yujie L and Yunchao H: MicroRNA-195 inhibits non-small cell lung cancer cell proliferation, migration and invasion by targeting MYB. Cancer Lett 347: 65-74, 2014.

30. Wei P, Zhang N, Wang Y, Li D, Wang L, Sun X, Shen C, Yang Y, Zhou X and Du X: FOXM1 promotes lung adenocarcinoma invasion and metastasis by upregulating SNAIL. Int J Biol Sci 11: $186-198,2015$

31. Guittat L, Alberti P, Gomez D, De Cian A, Pennarun G, Lemarteleur T, Belmokhtar C, Paterski R, Morjani H, Trentesaux C, et al: Targeting human telomerase for cancer therapeutics. Cytotechnology 45: 75-90, 2004.

32. Downward J: Targeting RAS signalling pathways in cancer therapy. Nat Rev Cancer 3: 11-22, 2003.

33. Sebolt-Leopold JS: Advances in the development of cancer therapeutics directed against the RAS-mitogen-activated protein kinase pathway. Clin Cancer Res 14: 3651-3656, 2008.

34. Longley DB, Harkin DP and Johnston PG: 5-fluorouracil: Mechanisms of action and clinical strategies. Nat Rev Cancer 3: 330-338, 2003.

35. Takada M, Fukuoka M, Kawahara M, Sugiura T, Yokoyama A Yokota S, Nishiwaki Y, Watanabe K, Noda K, Tamura T, et al: Phase III study of concurrent versus sequential thoracic radiotherapy in combination with cisplatin and etoposide for limited-stage small-cell lung cancer: Results of the Japan clinical oncology group study 9104. J Clin Oncol 20: 3054-3060, 2002.
36. Lü JM, Yao Q and Chen C: Ginseng compounds: An update on their molecular mechanisms and medical applications. Curr Vasc Pharmacol 7: 293-302, 2009

37. Chen X, Xie D, Wang L, Zhao Q, You ZH and Liu H: BNPMDA: BNPMDA: Bipartite network projection for MiRNA-disease association prediction. Bioinformatics 34: 3178-3186, 2018

38. Chen X, Yan CC, Zhang X and You ZH: Long non-coding RNAs and complex diseases: From experimental results to computational models. Brief Bioinform 18: 558-576, 2017.

39. Jiang W, Mitra R, Lin CC, Wang Q, Cheng F and Zhao Z: Systematic dissection of dysregulated transcription factor-miRNA feed-forward loops across tumor types. Brief Bioinform 17: 996-1008, 2016.

This work is licensed under a Creative Commons Attribution-NonCommercial-NoDerivatives 4.0 International (CC BY-NC-ND 4.0) License. 